
Not for reproduction or distribution or commercial use.

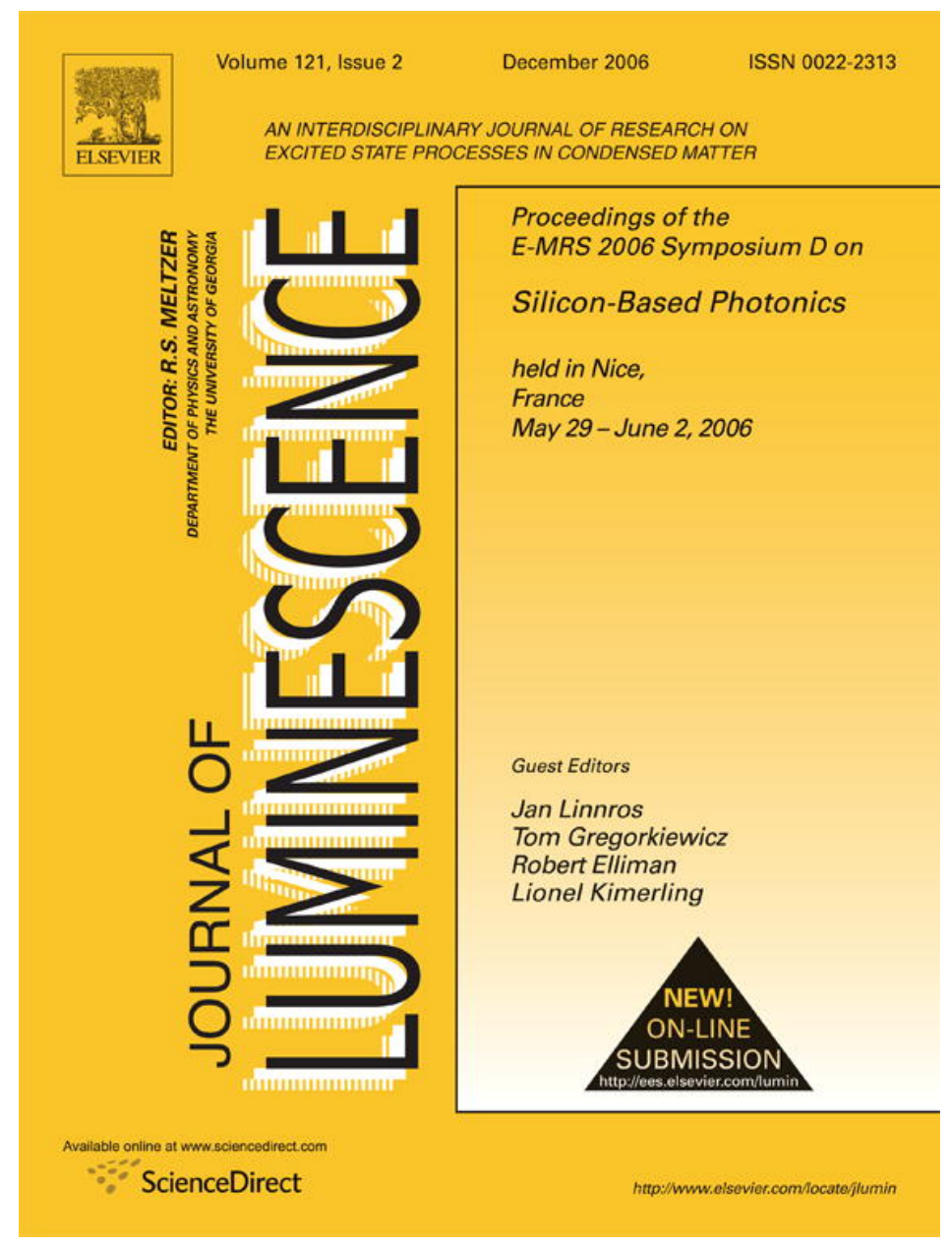

This article was originally published in a journal published by Elsevier, and the attached copy is provided by Elsevier for the author's benefit and for the benefit of the author's institution, for non-commercial research and educational use including without limitation use in instruction at your institution, sending it to specific colleagues that you know, and providing a copy to your institution's administrator.

All other uses, reproduction and distribution, including without limitation commercial reprints, selling or licensing copies or access,

or posting on open internet sites, your personal or institution's website or repository, are prohibited. For exceptions, permission may be sought for such use through Elsevier's permissions site at: 


\title{
The non-linear refractive index of colloidal PbSe nanocrystals: Spectroscopy and saturation behaviour
}

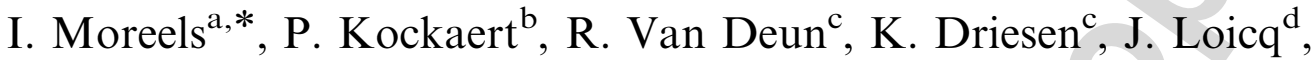 \\ D. Van Thourhout ${ }^{\mathrm{e}}$, Z. Hens ${ }^{\mathrm{a}}$ \\ a Physical Chemistry Laboratory, Ghent University, Krijgslaan 281-S12, B-9000 Ghent, Belgium \\ ${ }^{\mathrm{b}}$ Optics and Acoustics Department, Université Libre de Bruxelles, Av. Adolphe Buyl 87, B-1050 Brussels, Belgium \\ ${ }^{\mathrm{c}}$ Department of Chemistry, Katholieke Universiteit Leuven, Celestijnenlaan 200F, B-3001 Heverlee, Belgium \\ ${ }^{\mathrm{d}}$ Centre Spatial de Liège, Université de Liège, Sart-Tilman Scientifique Park, B-4031 Angleur, Belgium \\ ${ }^{\mathrm{e}}$ Department of Information Technology, Ghent University-IMEC, Sint-Pietersnieuwstraat 41, B-9000 Ghent, Belgium
}

Available online 22 September 2006

\begin{abstract}
A spectroscopic study of the optical non-linearities of PbSe colloidal solutions was performed with the $Z$-scan technique, at wavelengths between 1200 and $1750 \mathrm{~nm}$. No non-linear absorption is observed, while the third-order non-linear refractive index $n_{2}$ shows clear resonances, somewhat blueshifted relative to the exciton transitions in the absorbance spectrum. The occurrence of thermal effects is ruled out by time-resolved measurements. At resonance, measured $n_{2}$ values exceed typical bulk semiconductor values by two orders of magnitude. At high optical intensity, the refractive index change saturates, indicating that state filling lies at the origin of the observed effect.
\end{abstract}

(C) 2006 Elsevier B.V. All rights reserved.

Keywords: Non-linear optics; Quantum dots; Z-scan; PbSe

\section{Introduction}

All-optical signal processing (spectral filtering, optical switching, etc.) requires materials with a large third-order non-linear susceptibility $\chi^{(3)}$, or equivalently a large nonlinear refractive index $n_{2}$. At telecom wavelengths $(\lambda=1.55 \mu \mathrm{m})$, typical bulk semiconductors have only a small non-linear refractive index [1] (silicon: $n_{2}=0.45 \times$ $10^{-13} \mathrm{~cm}^{2} / \mathrm{W}$, GaAs: $n_{2}=1.59 \times 10^{-13} \mathrm{~cm}^{2} / \mathrm{W}$ ), so they are not very suitable to achieve a strong optical Kerr effect. To overcome these problems and implement all-optical devices on a semiconductor platform, one could use a hybrid approach, in which materials with a strong $n_{2}$ are integrated as the active element. One promising class of materials consists of colloidal nanocrystals.

Unlike their bulk counterparts, the electrical and optical properties of colloidal nanocrystals (or quantum dots) can be effectively engineered over a wide spectral range by

\footnotetext{
*Corresponding author. Tel.: + 3292644865; fax: + 3292644971.

E-mail address: Iwan.Moreels@UGent.be (I. Moreels).
}

tailoring the size of the particles. At present, colloidal chemistry techniques offer a variety of materials and sizes, producing particle dispersions of uniform size with optical properties ranging from the visible spectrum (e.g., with CdSe nanocrystals [2]) to the mid-infrared (e.g., with $\mathrm{PbSe}$ nanocrystals [3]). As the particles are dispersed in a solvent, processing of the nanocrystals offers a great flexibility. This has already resulted in the application of colloidal nanocrystals in opto-electronic and photovoltaic devices (photodetectors [4], LEDs [5] and lasers [2]).

Photonic devices consist of a new range of potential applications. Here we not only focus on the efficient generation of light but also on the manipulation of light, taking advantage of unique optical properties of semiconductor quantum dots. Recent studies have already demonstrated that quantum dots show an enhanced nonlinear refractive index [6] and behave as saturable absorbers [7,8]. These effects can be used for instance to achieve all-optical switching [9]. Unfortunately, most experiments that have investigated the non-linear optical properties of colloidal nanocrystals are either performed 
far from the bandgap $[6,8]$, or have yielded results cloaked by thermal effects when performed around the bandgap $[7,10]$.

Here we present the results of a detailed spectroscopic study of the non-linear refractive index $n_{2}$ of colloidal $\mathrm{PbSe}$ nanocrystals $(\mathrm{Q}-\mathrm{PbSe})$, in the near-infrared wavelength region $(1200-1750 \mathrm{~nm})$. Using different particle sizes between 3.9 and $5.8 \mathrm{~nm}$, we measured the non-linear refractive index $n_{2}$ with the $Z$-scan technique as a function of wavelength and optical intensity, covering both the transparent region and wavelengths near and above the bandgap. We found a large resonant $n_{2}$, with values up to -3 to $-4 \times 10^{-11} \mathrm{~cm}^{2} / \mathrm{W}$ for ca. $1 \mu \mathrm{M}$ solutions. At high optical intensities a clear saturation of the change in nonlinear refractive index $\delta n=n_{2} I_{0}$ is observed, but the saturation intensity lies well above the theoretical single exciton-saturation intensity. This may indicate that the non-linear refractive index observed is mainly due to biexcitons present in the Q-PbSe.

\section{Experimental}

State-of-the-art wet chemical synthesis is currently capable to yield Q-PbSe particle dispersions with sizes varying from 2 to $16 \mathrm{~nm}$, all with size distributions of less than $5 \%$ [3,11]. We adapted the synthesis described by Murray et al. [11] to produce monodisperse $(\sigma<5 \%)$ Q-PbSe, capped with oleic acid. Typically two precursor solutions are prepared: the first contains $0.19 \mathrm{~g}$ lead acetate in $3.15 \mathrm{ml}$ diphenylether (DPE) and $640 \mu \mathrm{l}$ oleic acid, the second $0.11 \mathrm{~g}$ selenium in $1.43 \mathrm{ml}$ trioctylphosphine (TOP). These precursor solutions are first heated to $130{ }^{\circ} \mathrm{C}$ for an hour, then allowed to cool down to room temperature and subsequently mixed and rapidly injected in DPE at $160{ }^{\circ} \mathrm{C}$. The temperature drops to $120^{\circ} \mathrm{C}$, at which the reaction is allowed to continue for $1-15 \mathrm{~min}$, where longer growth times yield larger nanocrystals. Hereafter, the reaction is quenched with an excess of butanol and methanol. After centrifugation, the dry powder containing PbSe nanocrystals of uniform size can be redispersed in any apolar solvent of choice.

To perform our $Z$-scan experiments, three different samples were prepared (see Table 1). Sizes and size distributions were determined from absorbance data using

Table 1

Size and concentration of the three samples prepared

\begin{tabular}{llll}
\hline Sample & Size $(\mathrm{nm})$ & Conc. $(\mu \mathrm{M})$ & $\lambda_{0}(\mathrm{~nm})$ \\
\hline A & 5.83 & 0.53 & 1693 \\
B & 5.21 & 0.86 & 1555 \\
C & 3.93 & 1.10 & 1245 \\
\hline
\end{tabular}

$\lambda_{0}$ denotes the wavelength of the first one-photon transition. Sizes and size distributions were determined using an experimental sizing curve. Concentrations were determined by weighing the carefully dried samples, taking the weight of the capping molecules into account. an experimental sizing curve (Fig. 1), based on different literature sources [3,11-17]. The sizing curve correlates the bandgap $E_{\mathrm{g}}$ of the nanocrystals with the mean size $d$ of the Q-PbSe dispersion

$$
E_{\mathrm{g}}=0.278+\frac{1}{0.014 d^{2}+0.29 d+0.036} \text {. }
$$

The size distribution is determined from the heterogeneous linewidth of the first absorbance peak. Concentrations are determined by weighing the thoroughly dried samples, taking the weight of the oleic acid capping molecules into account. To exclude non-linear effects from the solvent, the particles were dispersed in liquid $\mathrm{CCl}_{4}$, a transparent and weakly non-linear solvent at NIR wavelengths.

The non-linear refractive index $n_{2}$ and non-linear absorption $\beta$ were determined with the $Z$-scan technique [18], using a tunable femtosecond-mode-locked laser operating at an $82 \mathrm{MHz}$ repetition rate and at a wavelength in the intervals $1200-1350 \mathrm{~nm}$ and $1540-1750 \mathrm{~nm}$. As the calculation of $n_{2}$ relies on the shape of the laser pulses, beam profiling is an important aspect. Using an optical auto-correlator, we measured a $\operatorname{sech}^{2}$ temporal pulse profile. The pulse duration $\tau_{\mathrm{p}}$ varied between 75 and $90 \mathrm{fs}$, resulting in a spectral linewidth of $18-24 \mathrm{~nm}$ (fullwidth at half-maximum). The spatial profile can be determined by measuring the temporal buildup of the beam intensity as a chopper slit passes through the beam. From the derivative of the resulting curve, we could conclude the beam had a Gaussian shape. The beam waist at the focus of the Gaussian beam $w_{0}(61 \mu \mathrm{m})$ and corresponding Rayleigh length $z_{\mathrm{R}}(7.8 \mathrm{~mm})$, remained constant throughout the spectral region studied, with deviations occurring only near the edges (at 1200 and $1750 \mathrm{~nm}$ ). Based on the noise levels of our detectors, the thresholds of our setup (measured at an on-axis optical intensity $\left.I_{0}=100 \mathrm{MW} / \mathrm{cm}^{2}\right)$ corresponded to $n_{2}=$ $10^{-13} \mathrm{~cm}^{2} / \mathrm{W}$ and $\beta=0.5 \mathrm{~cm} / \mathrm{GW}$. The $n_{2}$-spectroscopy is measured at a fixed optical intensity $I_{0}=12 \mathrm{MW} / \mathrm{cm}^{2}$. Measuring in a wavelength interval of 1200-1750 nm using three different Q-PbSe samples, we covered a spectral range from full transparency up to the second exciton transition. At a fixed wavelength, we also performed time-resolved measurements. Using a $400 \mathrm{~Hz}$ chopper (50\% duty cycle) to produce $1.25 \mathrm{~ms}$ pulse trains, we recorded the temporal on-axis response with an oscilloscope. To investigate the intensity dependence of the nonlinear refractive index, we measured the change in refractive index $\delta n$ at optical intensities ranging from 0.74 to $84 \mathrm{MW} / \mathrm{cm}^{2}$.

\section{Results and discussion}

Measurements on pure $\mathrm{CCl}_{4}$ show a flat $Z$-scan trace, implying $n_{2}$ resp. $\beta$ fall below the detection threshold. Fig. 2b shows a typical $Z$-scan trace, measured on sample A $\left(I_{0}=11.1 \mathrm{MW} / \mathrm{cm}^{2}, \lambda=1640 \mathrm{~nm}\right)$. The flat open-aperture 


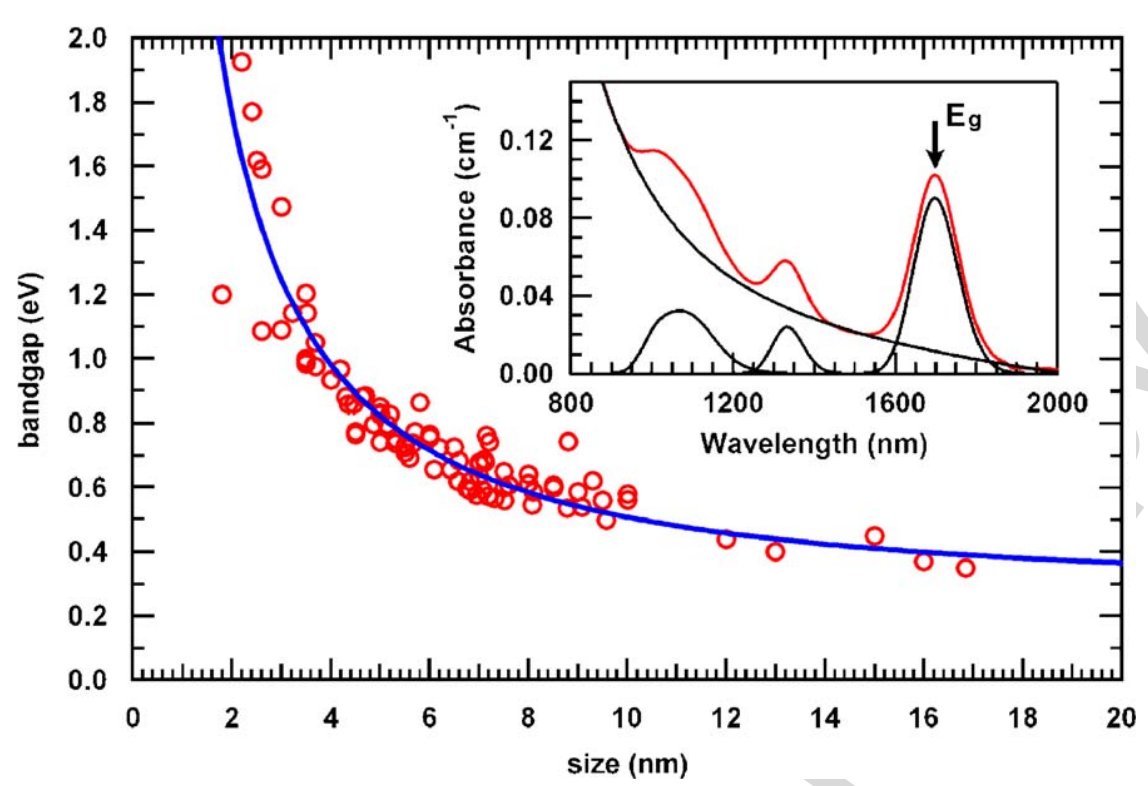

Fig. 1. Sizing curve, plotting the bandgap $E_{\mathrm{g}}$ of the $\mathrm{Q}-\mathrm{PbSe}(\mathrm{eV})$ as a function of the size $d$ of the particles (nm). Inset: absorbance spectrum of sample $\mathrm{A}$. Three absorbance peaks can clearly be observed, attributable to the $1 S_{\mathrm{e}}-1 S_{\mathrm{h}}, 1 P_{\mathrm{e}}-1 P_{\mathrm{h}}$ and $1 D_{\mathrm{e}}-1 D_{\mathrm{h}}$ transitions [17].
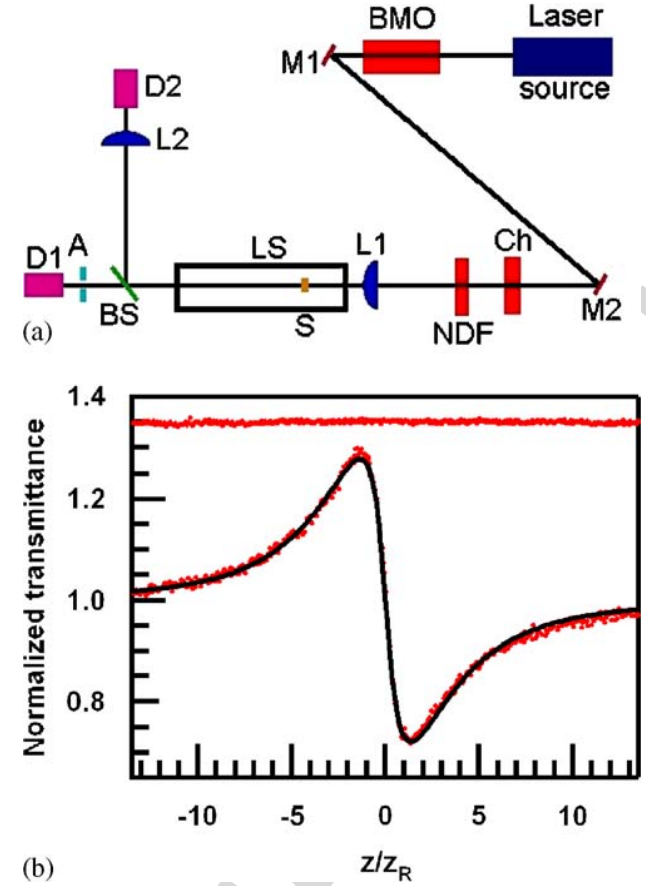

Fig. 2. (a) Experimental $Z$-scan setup. Mirrors $M 1$ and $M 2$ align the laser beam. Beam-massaging optics (BMO) ensure a nearly circular Gaussian beam. The linear stage (LS) translates the sample (S) along the path of the focused beam. Using a beam splitter, the on-axis transmittance is measured with detector D1 using an aperture (closed aperture $Z$-scan), while D2 collects the entire beam with lens L2 (open aperture $Z$-scan). A chopper $(\mathrm{Ch})$ is used for the time-resolved measurements, and neutraldensity filters (NDF) enable us to adjust the optical intensity. (b) Typical $Z$-scan trace. The open-aperture trace (top curve) shows there is no significant non-linear absorption. The peak-valley shape of the bottom trace (closed aperture) indicates a strong, negative non-linear refractive index. trace (top) indicates that the non-linear absorption falls below the detection threshold of $4.5 \mathrm{~cm} / \mathrm{GW}$ (the higher threshold results from the lower optical intensity $I_{0}$ used). Since we never observed non-linear absorption, we will only refer to the non-linear refractive index from here on. From the strong signal in the closed-aperture trace (bottom), we can immediately conclude that we have a large negative onaxis phase shift $\Delta \Phi_{0}$. The first-order function generally used to fit closed-aperture $Z$-scan data is valid only for small $\Delta \Phi_{0}$. We therefore expanded this expression up to the third order in $\Delta \Phi_{0}$, enabling us to accurately determine phase shifts up to $\Delta \Phi_{0}<1.75$

$$
\begin{aligned}
T(x)= & +\frac{4 x \Delta \Phi_{0}}{\left(1+x^{2}\right)\left(9+x^{2}\right)}+\frac{4\left(3 x^{2}-5\right) \Delta \Phi_{0}^{2}}{\left(1+x^{2}\right)^{2}\left(9+x^{2}\right)\left(25+x^{2}\right)} \\
& +\frac{32\left(x^{2}-11\right) x \Delta \Phi_{0}^{3}}{\left(1+x^{2}\right)^{3}\left(9+x^{2}\right)\left(25+x^{2}\right)\left(49+x^{2}\right)}
\end{aligned}
$$

with $x=z / z_{\mathrm{R}}$ the normalized distance from the focus. Upon fitting the experimental $Z$-scan traces, we noticed that they contained significant broad wings, not accounted for by the theoretical fit. These wings only appeared when measuring in the wavelength interval 1540-1750 nm (idler exit of the laser), and they were dependent on the laserbeam alignment. We therefore attribute these features to the laser setup, and take them into account by adding a second term to the equation

$T_{1}(x)=1+\frac{4 x z_{\mathrm{R}} / z_{1} \Delta \Phi_{1}}{\left(1+\left(x z_{\mathrm{R}} / z_{1}\right)^{2}\right)\left(9+\left(x z_{\mathrm{R}} / z_{1}\right)^{2}\right)}$.

The validity of this approach was confirmed by the results of the fit when allowing the Rayleigh length to be varied: only when using $T(x)+T_{1}(x)$ the fit yielded the 
correct $z_{\mathrm{R}} \cdot z_{1}$ typically varied between 3 and 3.5 times $z_{\mathrm{R}} \cdot n_{2}$ can be calculated from $\Delta \Phi_{0}=(2 \pi) /\left(\lambda n_{2} I_{0} L_{\text {eff }}\right)$, with $L_{\text {eff }}=(1-\exp (-\alpha L)) / \alpha$ the effective sample length. Due to the low absorbance $\alpha$, this corresponds to the true sample length $L$. Using this expression, we obtained $n_{2}=-3.0 \times$ $10^{-11} \mathrm{~cm}^{2} / \mathrm{W}$ for the results of Fig. $2 \mathrm{~b}$.

Fig. 3 represents the temporal response of the on-axis intensity, measured on sample A at $\lambda=1693 \mathrm{~nm}$ (maximal absorbance). When there is no sample present (NS) the trace shows a fast signal buildup of $43 \mu \mathrm{s}$. This rise time is due to the finite beam waist and corresponds to the time the chopper needs to pass through the beam. When placing the sample far from the focus of the beam (FF) or at the position where the closed-aperture trace reaches its maximum (OR), we observe identical traces except a scaling factor. Thermal effects, with a rise time $t_{\mathrm{c}}=$ $w_{0}^{2} /(4 D)$ ( $D$ the thermal diffusivity of $\mathrm{CCl}_{4}$ ) of $12 \mathrm{~ms}$, would be clearly distinguishable in our measurements. From these results we conclude that thermal lensing does not contribute to the non-linear refractive index.

Having established the electronic nature of the nonlinear refractive index, we measured $n_{2}$ as a function of wavelength (Fig. 4). In the $n_{2}$-spectrum of sample A we see two bell-shaped resonances, comparable in width to the exciton peaks of the absorbance spectrum, but somewhat blueshifted. Measurements on sample B and sample $\mathrm{C}$ yield similar results. At energies far below the first exciton transition, $n_{2}$ drops below the detection threshold. The small peak observed in sample $\mathrm{C}$ is probably due to oleic acid present in the sample. These results demonstrate that the value of the non-linear refractive index can be optimized at any NIR wavelength by choosing the appropriate size of Q-PbSe. Maximal $n_{2}$-values correspond to -3 to $-4 \times 10^{-11} \mathrm{~cm}^{2} / \mathrm{W}$ for ca. $1 \mu \mathrm{M}$ solutions, exceeding typical bulk semiconductor values [1] (Si and $\mathrm{GaAs}$ ) at these wavelengths by two orders of magnitude. As the measured $n_{2}$ pertains to dilute Q-PbSe solutions, it increases linearly with the Q-PbSe volume fraction $f: n_{2, \text { eff }}=f n_{2, \mathrm{PbSe}}$. Determination of $n_{2}$ for different concentrations of sample $A$ at $\lambda=1640 \mathrm{~nm}$ yields $n_{2, \mathrm{PbSe}}=-6.5 \times 10^{-7} \mathrm{~cm}^{2} / \mathrm{W}$, which is among the highest values reported for semiconductor nanocrystals $[6-8,10]$.

Fig. 5c shows the change of refractive index $\delta n$ when the optical intensity is varied from 0.74 to $84 \mathrm{MW} / \mathrm{cm}^{2}$, measured on sample $\mathrm{A}$ at $\lambda=1640 \mathrm{~nm}$ (maximal $n_{2}$ ). At low optical intensities, $n_{2}$ increases linearly with $I_{0}$, as expected for a third-order non-linearity. However, as the optical intensity increases, we observed a saturation of the change in refractive index $\delta n$. This saturation is taken into account by following model for $\delta n$ :

$\delta n=\frac{n_{2} I_{0}}{1+I_{0} / I_{\mathrm{s}, \mathrm{ex}}}$

with $I_{\mathrm{s}, \mathrm{ex}}$ the experimental saturation intensity. Fitting the data yields $I_{\mathrm{s}, \mathrm{ex}}=39.6 \mathrm{MW} / \mathrm{cm}^{2}$.

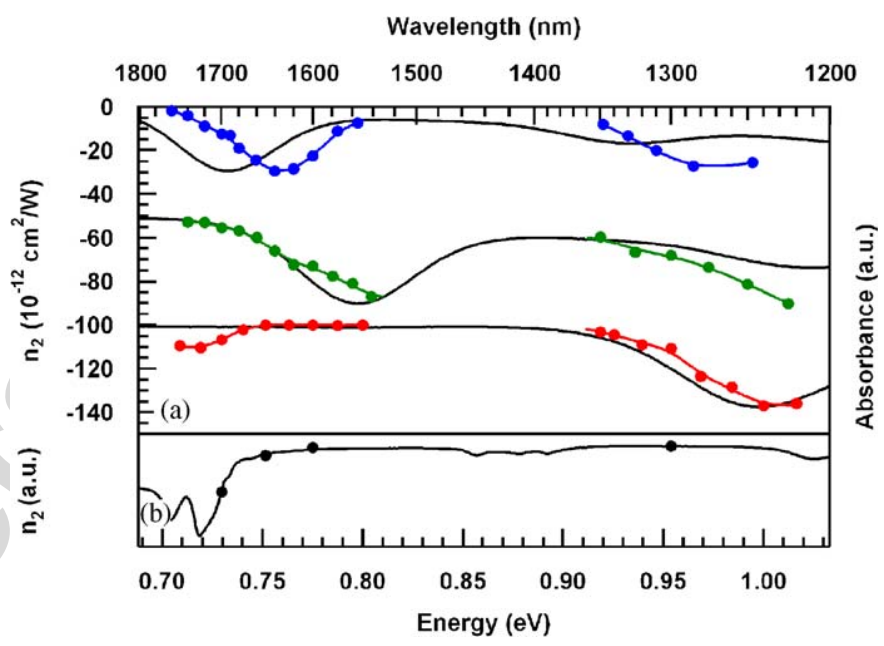

Fig. 4. (a) Spectroscopy of the non-linear refractive index of Q-PbSe of sample A (top, blue curve), B (middle, green) and C (bottom, red), measured at $I_{0}=12 \mathrm{MW} / \mathrm{cm}^{2}$. The dots and interpolated curve show $n_{2}$, the full line shows the absorbance spectrum. A bell-shaped resonance is observed for $n_{2}$, with a width comparable to the width of the exciton peaks of the absorbance spectrum (black curve). The small peak observed in sample $\mathrm{C}$ is due to the oleic acid attached to the Q-PbSe surface. (b) $n_{2-}$ spectrum of a $63.4 \mathrm{mM}$ oleic acid solution in $\mathrm{CCl}_{4}$. The dots represent the non-linear refractive index, the full line shows the absorbance spectrum.
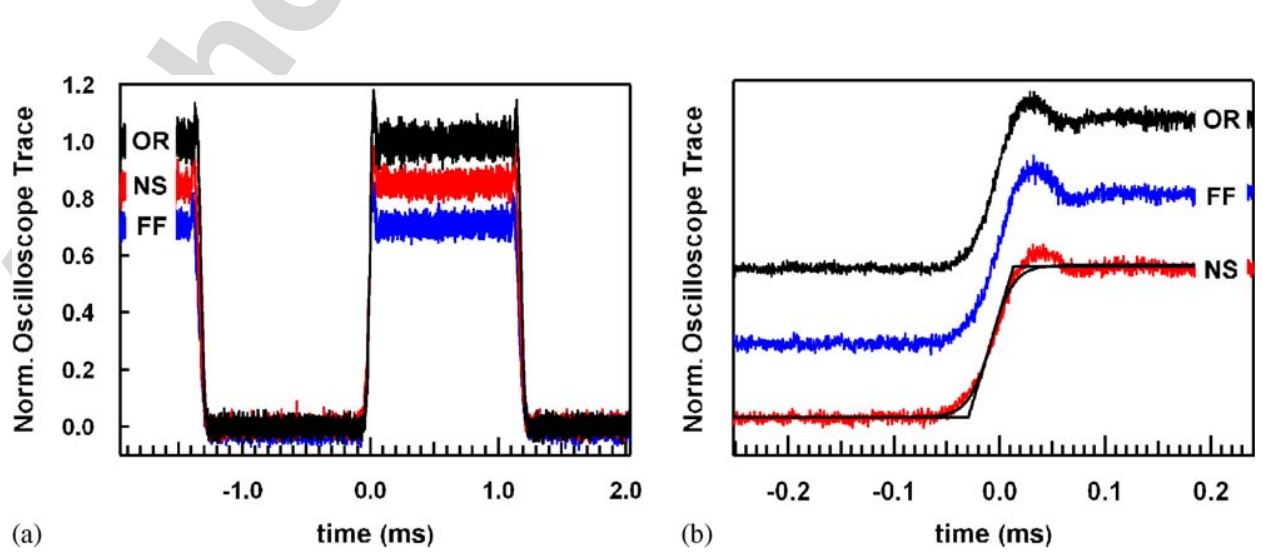

Fig. 3. Results of the time-resolved measurements, performed on sample A at $\lambda=1693 \mathrm{~nm}$. (a) Measurements without the sample (NS), with the sample far from the focus of the beam (FF) and at $z=-1.2 z_{\mathrm{R}}(\mathrm{OR})$ yield identical results, except a scaling factor due to $n_{2}$. (b) All three traces show the same fast buildup ( $43 \mu \mathrm{s})$, excluding thermal lensing as the origin of the observed $n_{2}$. 

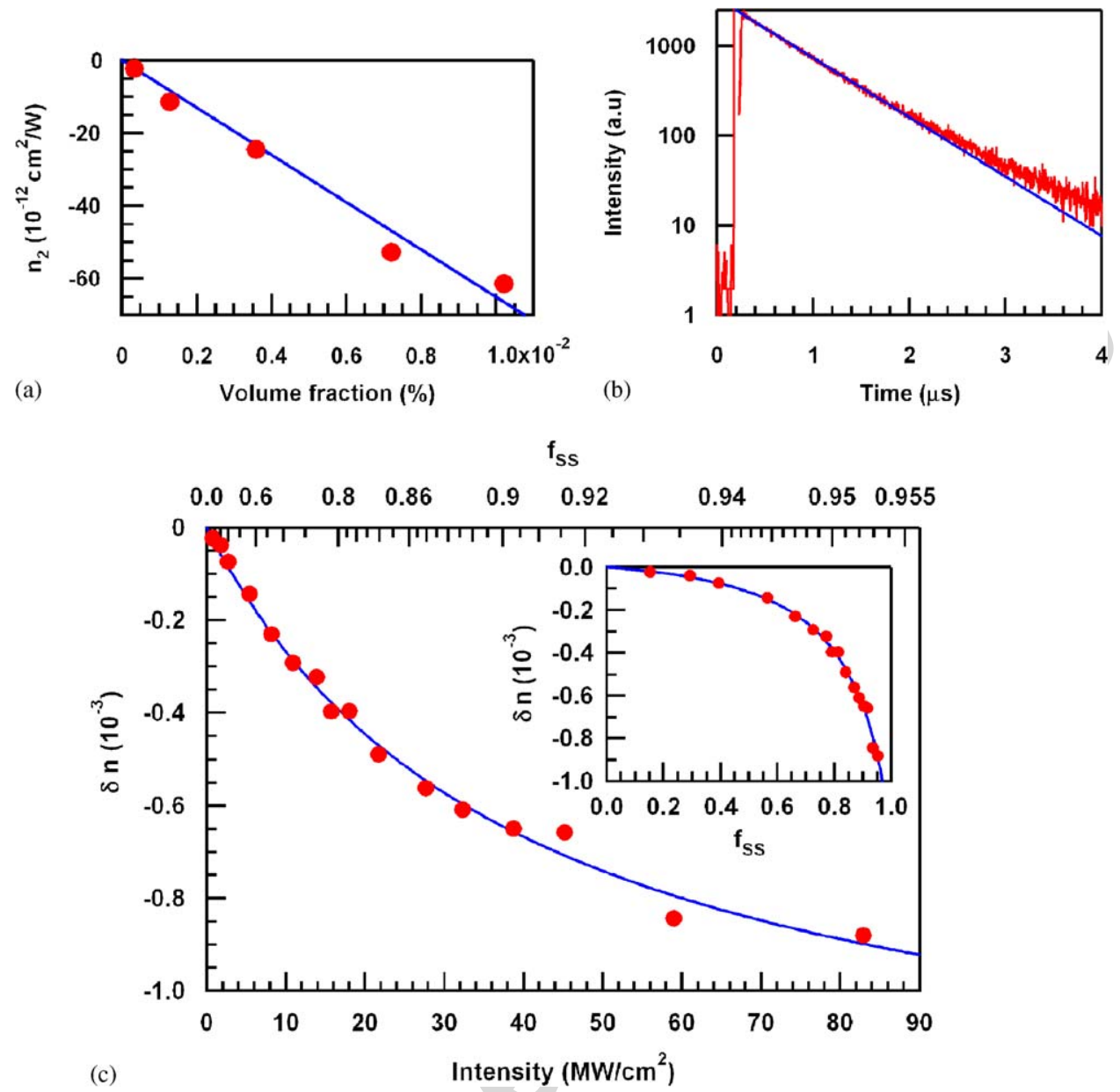

Fig. 5. (a) Measured $n_{2}$ for different volume fractions of the nanocrystals (sample A, $\lambda=1640 \mathrm{~nm}$ ). We see a linear increase of $n_{2}$ with the Q-PbSe concentration, as expected for dilute dispersions. (b) Time-resolved luminescence decay of $5.4 \mathrm{~nm} \mathrm{Q}-\mathrm{PbSe}$. The decay time corresponds to $0.66 \mu$ s. (c) Measured $\delta n$ as a function of the optical intensity $I_{0}$. The experimental saturation intensity corresponds to $39.6 \mathrm{MW} / \mathrm{cm}^{2}$. The top axis shows the respective fraction of excited nanocrystals $f_{\mathrm{SS}}$. Inset: a strong increase of $\delta n$ is observed, even when $f_{\mathrm{SS}}$ approaches unity. This suggests that bi-excitons contribute substantially to the non-linear refractive index.

This saturation indicates that state filling lies at the origin of the observed non-linear refractive index. Theoretically, around the first exciton transition a quantum confined PbSe nanocrystal can be described as a two-level system. We calculated the fraction of excited nanocrystals $f$ (Q-PbSe containing an exciton) as a function of optical intensity and determined the corresponding saturation intensity $I_{\mathrm{s}, \text { th }}$. Time-resolved luminescence measurements have revealed that the exciton lifetime is several orders of magnitude larger than the time between pulses $1 / v=12.2 \mathrm{~ns}$. For $5.4 \mathrm{~nm}$ particles, we measured a lifetime $\tau_{\text {ex }}=0.66 \mu$ s (Fig. 5b). The high pulse rate will therefore cause a buildup of excited nanocrystals until a steady-state fraction $f_{\mathrm{SS}}$ is reached. Denoting $c^{\mathrm{o}}$ the concentration of unexcited Q-PbSe and $c^{*}$ the concentration of excited Q-PbSe, we can define $f_{\text {SS }}$ as

$f_{\mathrm{SS}}=\frac{c^{*}}{c^{\mathrm{o}}+c^{*}}$.

Taking the pulse duration $\tau_{\mathrm{p}}$ and spectral width $\lambda_{\mathrm{p}}$ into account, the number of nanocrystals excited per pulse is given by the ratio of the absorbed energy $E_{\mathrm{abs}}$ and the maximal energy $F E_{\mathrm{g}}$ one mole Q-PbSe can absorb

$N_{\text {exc }}=\frac{E_{\text {abs }}}{F E_{\mathrm{g}}}=\frac{\ln (10) \varepsilon c^{\circ} L \tau_{\mathrm{p}} I_{0}}{F E_{\mathrm{g}} \lambda_{\mathrm{p}}}$,

where $L$ denotes the length of the sample, $\varepsilon$ is the molar extinction coefficient of the nanocrystals. The number of $\mathrm{Q}-\mathrm{PbSe}$ relaxing to the ground state in between pulses corresponds to $\left(\nu \tau_{\mathrm{ex}} \gg 1\right)$

$N_{\mathrm{des}}=c^{*} L\left(1-\exp \left(-\frac{1}{v \tau_{\mathrm{ex}}}\right) \approx \frac{c^{*} L}{v \tau_{\mathrm{ex}}}\right.$.

In steady state, the number of excitations per pulse equals the number of de-excitations in between pulses, resulting in a steady-state fraction of excited nanocrystals

$f_{\mathrm{SS}}=\frac{\ln (10) \varepsilon \tau_{\mathrm{p}} v \tau_{\mathrm{ex}} I_{0}}{F E_{\mathrm{g}} \lambda_{\mathrm{p}}+\ln (10) \varepsilon \tau_{\mathrm{p}} v \tau_{\mathrm{ex}} I_{0}}$

with corresponding saturation intensity

$I_{\mathrm{s}, \mathrm{th}}=\frac{F E_{\mathrm{g}} \lambda_{\mathrm{p}}}{\ln (10) \varepsilon \tau_{\mathrm{p}} v \tau_{\mathrm{ex}}}$. 
This gives an expected saturation intensity of $4.0 \mathrm{MW}$ $\mathrm{cm}^{2}$, an order of magnitude lower than the experimentally observed one. It indicates that the fraction of excited nanocrystals is high even at low optical intensity. But more importantly, the experimental $\delta n$ still increases markedly, even at high $f_{\mathrm{SS}}$ (inset Fig. 5c). This large difference between the expected and the experimental saturation intensity may indicate that the creation of bi-excitons in already excited nanocrystals contributes significantly to the non-linear refraction. The creation of bi-excitons at high optical intensities has already been observed with Q-PbSe, using time-resolved optical bleaching [19]. These measurements demonstrate that at high optical intensities, the bleaching shows a two-component decay: a fast, picosecond component due to the decay of bi-excitons and a slow component due to the decay of single excitons.

\section{Conclusions}

We presented a very detailed spectroscopic and intensitydependent study on the non-linear refractive index of dilute suspensions of PbSe nanocrystals. The results highlight the unique optical properties of colloidal quantum dots. The $n_{2}$-spectrum shows distinct bell-shaped resonances, slightly blue-shifted to the peaks in the absorbance spectrum. As these resonances are dependent on the size of the Q-PbSe, the non-linear refractive index can be optimized for any desired wavelength in the technologically important NIR wavelength region. Maximal $n_{2}$-values are two orders of magnitude larger than typical bulk semiconductor values. Considering the convenient processing of colloidal nanocrystals, we believe integrating Q-PbSe on a $\mathrm{Si}$ platform could potentially lead to new low-loss non-linear applications. The presence of thermal non-linearities is excluded by time-resolved measurements. By varying the beam intensity, we demonstrated that state filling leads to a saturation of the non-linear refractive index. However, the experimental saturation intensity is larger than expected. The theoretically calculated value, based on the creation of a single exciton within the nanocrystals, yields $4.0 \mathrm{MW} /$ $\mathrm{cm}^{2}$, an order of magnitude smaller than the experimental saturation intensity. Considering these results, we suggest that the creation of bi-excitons might lead to the large nonlinearities observed.

\section{Acknowledgements}

We acknowledge the Institute for the Promotion of Innovation through Science and Technology in Flanders (IWT-Vlaanderen) for a scholarship (I.M.), and the Fund for Scientific Research Flanders (FWO-Vlaanderen) for a research grant (Z.H., 1.5.089.03). This work was also supported by the Belgian Science Policy Office under Grant IAP-V18 (P.K., D.v.T.).

\section{References}

[1] M. Dinu, F. Quochi, H. Garcia, Appl. Phys. Lett. 82 (18) (2003) 2954.

[2] H.J. Eisler, V.C. Sundar, M.G. Bawendi, M. Walsh, H.I. Smith, V. Klimov, Appl. Phys. Lett. 80 (24) (2002) 4614.

[3] J.M. Pietryga, R.D. Schaller, D. Werder, M.H. Stewart, V.I. Klimov, J.A. Hollingsworth, J. Am. Chem. Soc. 126 (38) (2004) 11752.

[4] D.F. Qi, M. Fischbein, M. Drndic, S. Selmic, Appl. Phys. Lett. 86 (9) (2005) 093103.

[5] J. Roither, W. Heiss, D.V. Talapin, N. Gaponik, A. Eychmuller, Appl. Phys. Lett. 84 (13) (2004) 2223.

[6] X.C. Wu, R.Y. Wang, B.S. Zou, P.F. Wu, L. Wang, J.R. Xu, W. Huang, Appl. Phys. Lett. 71 (15) (1997) 2097.

[7] I. Gerdova, A. Hache, Opt. Commun. 246 (1-3) (2005) 205.

[8] Y.C. Ker, J.H. Lin, W.F. Hsieh, Jpn. J. Appl. Phys. Part. 142 (3) (2003) 1258.

[9] R. Prasanth, J.E.M. Haverkort, A. Deepthy, E.W. Bogaart, J. van der Tol, E.A. Patent, G. Zhao, Q. Gong, P.J. van Veldhoven, R. Notzel, J.H. Wolter, Appl. Phys. Lett. 84 (20) (2004) 4059.

[10] J. Loicq, Y. Renotte, J.L. Delplancke, Y. Lion, New J. Phys. 6 (2004) 32 .

[11] C.B. Murray, S.H. Sun, W. Gaschler, H. Doyle, T.A. Betley, C.R. Kagan, IBM J. Res. Dev. 45 (1) (2001) 47.

[12] A. Lipovskii, E. Kolobkova, V. Petrikov, I. Kang, A. Olkhovets, T. Krauss, M. Thomas, J. Silcox, F. Wise, Q. Shen, S. Kycia, Appl. Phys. Lett. 71 (23) (1997) 3406.

[13] H. Du, C.L. Chen, R. Krishnan, T.D. Krauss, J.M. Harbold, F.W. Wise, M.G. Thomas, J. Silcox, Nano Lett. 2 (11) (2002) 1321.

[14] B.L. Wehrenberg, C.J. Wang, P. Guyot-Sionnest, J. Phys. Chem. B 106 (41) (2002) 10634

[15] J.S. Steckel, S. Coe-Sullivan, V. Bulovic, M.G. Bawendi, Adv. Mater. 15 (21) (2003) 1862.

[16] W.W. Yu, J.C. Falkner, B.S. Shih, V.L. Colvin, Chem. Mater. 16 (17) (2004) 3318.

[17] P. Liljeroth, P.A.Z. van Emmichoven, S.G. Hickey, H. Weller, B. Grandidier, G. Allan, D. Vanmaekelbergh, Phys. Rev. Lett. 95 (8) (2005) 086801.

[18] M. Sheikbahae, A.A. Said, T.H. Wei, D.J. Hagan, E.W. Vanstryland, IEEE J. Quantum Electron. 26 (4) (1990) 760.

[19] R.D. Schaller, M.A. Petruska, V.I. Klimov, J. Phys. Chem. B 107 (50) (2003) 13765 . 